\title{
Arthroscopic internal drainage and cystectomy of popliteal cyst in knee osteoarthritis
}

\author{
Jun Jiang $\mathbb{D}$ and Lei Ni
}

\begin{abstract}
Background: The purpose of this study was to evaluate the efficacy of arthroscopic knee cavity internal drainage and cyst cavity debridement operation of popliteal cyst in knee osteoarthritis patients.

Methods: From August 2007 to March 2013, 58 knee osteoarthritis patients with popliteal cyst were treated with arthroscopic knee cavity internal drainage through posteromedial portal and popliteal cyst cavity debridement through superior posteromedial portal. In all patients, preoperative magnetic resonance imaging (MRI) was performed to detect combined intra-articular pathology and the communication between popliteal cyst and knee cavity. Clinical efficacy was evaluated through VAS score and Lysholm score.

Results: All patients had neither recurrence of popliteal cyst nor complaints of pain, swelling, or functional impairment at average 24 months follow-up after surgery. Postoperatively, VAS score was decreased significantly and Lysholm score was raised significantly comparing preoperatively.

Conclusion: Arthroscopic knee cavity internal drainage operation through posteromedial portal and popliteal cyst cavity debridement through superior posteromedial portal is an effective minimally invasive surgery method for the treatment of popliteal cyst without recurrence in knee osteoarthritis patients.
\end{abstract}

Keywords: Popliteal cyst, Osteoarthritis, Knee, MRl, Arthroscopy

\section{Background}

A popliteal cyst, originally called Baker's cyst, is a synovial fluid-filled mass located in the popliteal fossa. The most common synovial popliteal cyst is considered to be a distension of the bursa located between the medial head of the gastrocnemius muscle and semimembranosus muscle. Usually, in an adult patient, popliteal cyst is complicated with knee osteoarthritis [1].

Some scholars thought that popliteal cyst in knee osteoarthritis patients is communicated with knee cavity and closely related to joint effusion [2]. When joint effusion is reduced, popliteal cyst will disappeared automatically and need not to be excised [3]. But our clinical experience shows that clinical result of conservative treatment for popliteal cyst in knee osteoarthritis patients is not good enough.

* Correspondence: 13601387336@163.com

Arthritis Clinic \& Research Center, Peking University People Hospital, \#11 Xizhimen South Avenue, Xicheng District, Beijing 100044, China
Usually, popliteal cyst in knee osteoarthritis patients will be excised through open surgery from posterior popliteal fossa by most orthopedic surgeon. Some scholars suggest that popliteal cyst can be arthroscopically excised directly from posterior popliteal fossa [4]. But both methods have high risk of injury to popliteal nerves and vessels and have high recurrence. Moreover, open surgery has cosmetic problems because of large incision scar [5].

Popliteal cysts have been shown to be complicated with knee osteoarthritis and have unidirectional synovial fluid valvular mechanism from knee cavity to popliteal cyst $[6,7]$. Arthroscopic knee cavity internal drainage (posteromedial capsulotomy between the medial head of gastrocnemius and semimembranosus muscle through posteromedial portal) will cause bidirectional synovial fluid flow mechanism between knee cavity and popliteal cyst. The correction of the unidirectional valvular mechanism would prevent recurrence of popliteal cyst. 
When joint effusion in knee osteoarthritis is reduced, popliteal cyst will shrink, even disappear gradually. So there is no need to perform open surgery. To facilitate popliteal cyst shrinking, arthroscopic cystectomy was also performed through superior posteromedial portal. Simultaneously, intra-articular lesion of knee osteoarthritis should be addressed and popliteal cyst cavity debridement through superior posteromedial portal should be performed arthroscopically.

The purpose of this study was to evaluate clinical efficacy of arthroscopic knee cavity internal drainage through posteromedial portal and cystectomy through superior posteromedial portal of popliteal cyst in knee osteoarthritis patients.

\section{Methods}

\section{Clinical data of patients}

From August 2007 to March 2013, 58 knee osteoarthritis patients with popliteal cyst were treated with arthroscopic knee cavity internal drainage through posteromedial portal and cystectomy through superior posteromedial portal. These patients include 18 men and 40 women. The average age was 63.5 years old (range $48 \sim 79$ years). These popliteal cysts were located in the left knee in 26 patients and in the right knee in 32 patients. The average course of disease was 24 months (range 18 60 months).

Inclusion criteria was primary osteoarthritis with popliteal cyst.

The exclusion criteria were as follows: posttraumatic knee osteoarthritis patients with popliteal cyst, knee rheumatic arthritis patients with popliteal cyst, inflammatory arthritis (gout, pseudogout, psoriasis) patients with popliteal cyst, and popliteal cyst patients after total knee arthroplasty.

To evaluate the intra-articular lesions, preoperative magnetic resonance imaging (MRI) was performed for all patients. MRI showed that popliteal cyst was located between the medial head of gastrocnemius and semimembranosus muscle and there was communication between popliteal cyst and knee cavity (Fig. 1).

The indication for the arthroscopic operation included an MRI-detected popliteal cystic lesion, mass swelling in the popliteal fossa, pain and limitation of ROM (range of motion), and symptoms associated with an intra-articular lesion of knee osteoarthritis.

\section{Surgical procedure}

These patients underwent arthroscopic surgery with spinal anesthesia in the supine position with tourniquet. Routine arthroscopic examination of the knee was performed through anterolateral (AL) portal. Arthroscopic debridement was performed to treat knee osteoarthritis including chondral lesion debridement, partial meniscectomy, and synovectomy through anteromedial (AM) portal. The arthroscope was redirected into posteromedial compartment from AL portal (or AM portal) through intercondylar notch between posterior cruciate ligament (PCL) and lateral wall of medial condyle with the knee at $60^{\circ}$ flexion. We observed the posteromedial capsule and inserted spinal needle into posteromedial articular cavity within the boundary composed of medial collateral ligament (MCL), medial gastrocnemius, and semimembranosus with knee at $90^{\circ}$ flexion, and then, we incised the skin and established a posteromedial (PM) portal. A probe was inserted to find the communication to the cyst. Usually, it is not easy to find the communication from articular cavity to the cyst. But there is always posteromedial capsular fold in front of popliteal cyst (Fig. 2a). The posteromedial capsular fold was resected with shaver through PM portal to thoroughly enlarge the communication between medial gastrocnemius tendon and semimembranosus tendon and to produce bidirectional flow of synovial fluid between knee cavity and popliteal cyst. The medial gastrocnemius tendon should be clearly revealed (Fig. 2b). Then, knee cavity internal drainage of popliteal cyst was accomplished arthroscopically. After this procedure, the arthroscope was transferred to
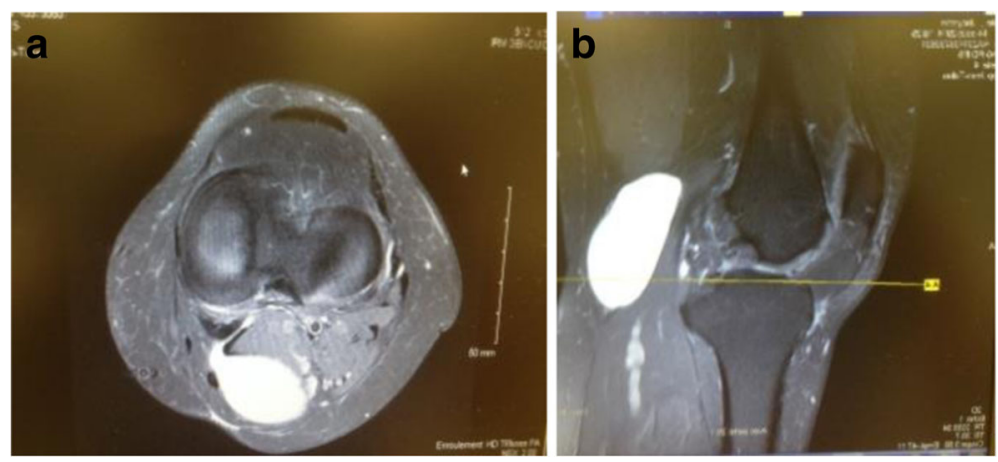

Fig. 1 Preoperative MRI image of the left knee popliteal cyst. a Axial view shows that the popliteal cyst is located between the medial gastrocnemius and semimembranosus, and there is communication between popliteal cyst and knee cavity. $\mathbf{b}$ Sagittal view shows that the popliteal cyst is located posteriorly to the medial gastrocnemius 

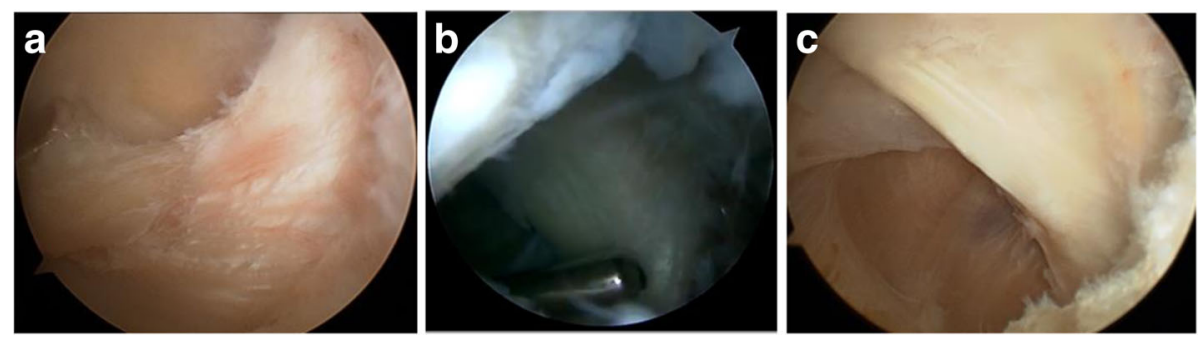

Fig. 2 a An arthroscopic view through anteromedial portal shows the posteromedial capsular fold in front of popliteal cyst in the left knee osteoarthritis patient. b An arthroscopic view through anteromedial portal shows knee cavity internal drainage of popliteal cyst through resecting posteromedial capsule between the medial gastrocnemius tendon and semimembranosus tendon to produce bidirectional flow of popliteal cyst synovial fluid between knee cavity and popliteal cyst. The medial gastrocnemius tendon can be clearly observed. c An arthroscopic view through posteromedial portal shows popliteal cyst cavity (posteromedial to the medial gastrocnemius tendon)

PM portal to view popliteal cyst cavity (Fig. 2c). Under the view, with the knee bent to figure 4, a superior posteromedial (SPM) portal was established. Through SPM portal, synovial membrane of popliteal cyst cavity was debrided to reveal the semimembrane tendon, gracilis tendon, and semitendinosus tendon from anterior to posterior (Fig. 3). This procedure is useful for adhesion of medial gastrocnemius tendon and semimembrane tendon to close popliteal cyst cavity. Associated intra-articular lesions, such as meniscus tear, chondral lesions, and synovitis, were then treated with corresponding arthroscopic procedure, such as meniscectomy, chondral lesion debridement, and synovectomy. Once a drain was inserted into the knee cavity through PM portal, a compressive dressing should be applied in the popliteal fossa. Operation time was about $30 \mathrm{~min}$ to $1 \mathrm{~h}$.

\section{Postoperative rehabilitation}

Patients were encouraged to perform active knee flexion and isometric quadriceps muscle strength exercises on the second day after surgery which should be performed for about 3 months postoperatively. The drain was removed 2 days after surgery. Then, the patients were allowed to take partial weight bearing with crutch for about 6 weeks. And full weight bearing was permitted 6 weeks postoperatively.

\section{Clinical evaluation}

In all patients, preoperative MRI was performed to detect intra-articular lesions and the communication between knee cavity and popliteal cyst. The patient was followed up postoperatively. We used VAS score and Lysholm score for clinical evaluation. The VAS score and Lysholm score were statistically compared between postoperatively and preoperatively with Student's $T$ test.

\section{Results}

\section{Symptoms and signs}

Preoperatively, popliteal cyst in knee osteoarthritis patients can cause discomfort and swelling symptoms. Some patients may feel weakness of the lower limb. When popliteal cyst was enlarged, it will influence range of motion. The knee joint could not be fully flexed or extended. The follow-up results postoperatively show that the aforementioned symptoms disappear.

Preoperatively, cystic mass, swelling, fluctuation, tension, and local tenderness in the popliteal fossa can be felt through physical examination. The follow-up

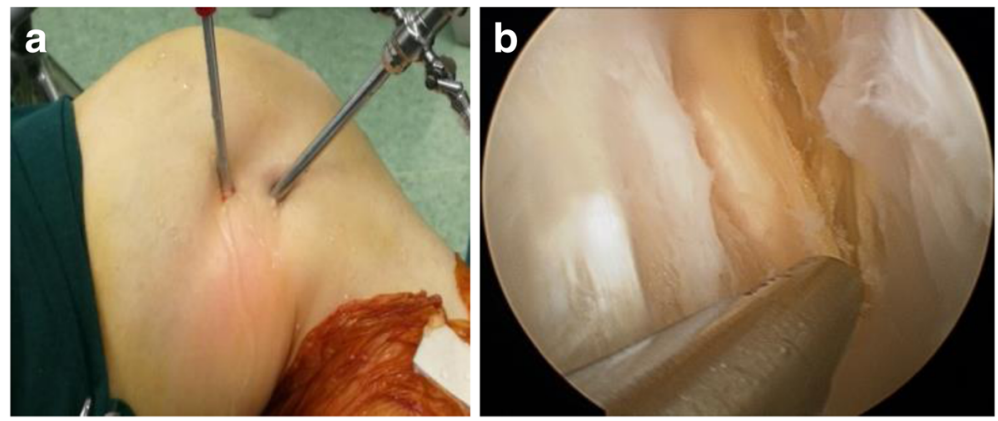

Fig. 3 a, b An arthroscopic view through posteromedial portal shows debridement of synovial membrane (on the right side of the shaver) of popliteal cyst cavity working through superior posteromedial portal. The semimembrane tendon, gracilis tendon, and semitendinosus tendon aligned from anterior to posterior on the left side of shaver 
results postoperatively show that the aforementioned signs disappear and no recurrence.

\section{Preoperative MRI examination}

Medial meniscus lesions were mostly found in 55 patients (grade III mild lesion). Chondral lesion in medial compartment and patellofemoral joint were totally found in all patients because of knee osteoarthritis. Some patients also had synovitis and hydrarthrosis.

\section{VAS pain score and Lysholm knee function score}

The follow-up period was average 24 months (18 60 months). Clinical efficacy of the surgical procedure was evaluated through VAS pain score and Lysholm knee function score. The VAS pain score was decreased from preoperative $8.8( \pm 3.5)$ points to postoperative $3.1( \pm 1.4)$ points. The Lysholm knee function score was raised from preoperative $43.6( \pm 5.3)$ points to $87.5( \pm 3.7)$ points. Both have statistical significance through Student's $T$ test, shown in Table 1.

\section{Discussion}

Knee osteoarthritis is a chronic degenerative disease in adults with the main pathology of cartilage degeneration and osteophyte, accompanied with meniscus tear, synovitis, and loose body. In adults, popliteal cysts usually occur concomitantly with knee osteoarthritis, which results in persistent and excess production of synovial fluid $[8,9]$.Through the study of 426 knees, Labropoulos et al. [10] found that the incidence rate of popliteal cyst increased with the age, especially for the population older than 50 years. Marti-Bonmati et al. [11] studied the prevalence of popliteal cyst through MRI examination. They found 145 popliteal cysts in 382 knees (38\%) and that there was extremely significant association between knee joint effusion and popliteal cyst $(P=0.002)$. There was a significant association between meniscal injury and popliteal cyst $(P=0.01)$.

Most scholars agree that popliteal cyst is a distention of the bursa located between the medial head of the gastrocnemius muscle and semimembranosus. The bursa space is communicated with knee cavity through one-way valvular mechanism covered by posteromedial capsular fold. Synovitis and chronical joint effusion in knee osteoarthritis will increase articular cavity pressure and can cause continuous unidirectional flow of synovial fluid from articular cavity to

Table 1 VAS and Lysholm score $(n=58 \quad \bar{X} \pm S)$

\begin{tabular}{lll}
\hline & VAS score & Lysholm score \\
\hline Preoperative & $8.8 \pm 3.5$ & $43.6 \pm 5.3$ \\
Postoperative & $3.1 \pm 1.4$ & $87.5 \pm 3.7$ \\
$T$ value & 10.96 & 78.53 \\
$P$ value & 0.0000 & 0.0000 \\
\hline
\end{tabular}

popliteal cyst because of low pressure in popliteal cyst. The synovial fluid in popliteal cyst could not return to knee articular cavity. So the popliteal cyst will be enlarged gradually.

Sansone and De Ponti [7] thought that medial meniscus lesion was the key of popliteal cyst formation. There was medial meniscus lesion in $84-90 \%$ of popliteal cyst patients. They thought that posterior horn tear of medial meniscus could act as valvular mechanism between knee articular cavity and popliteal cyst. But Takahashi and Nagano [12] had suspect on the theory. They put arthroscope into posteromedial compartment through posteromedial portal and found there was big distance between posterior horn of medial meniscus and communication of popliteal cyst with knee articular cavity. So it was impossible for posterior horn tear of the medial meniscus to act as valvular mechanism between knee articular cavity and popliteal cyst. Takahashi found "cranny structure" of posteromedial capsule observed in arthroscopic surgery can act as valvular mechanism.

According to our clinical data, there was no obvious "cranny structure" in posteromedial capsule. Maybe the "cranny structure" in posteromedial capsule is very tiny to observe arthroscopically. But posteromedial capsular fold can always be observed arthroscopically, which may be self-closure of communication between popliteal cyst and knee cavity to prevent its continuous enlargement.

There are two arthroscopic surgery methods to disrupt unidirectional flow of popliteal cyst in knee osteoarthritis patients. Communication enlargement is posteromedial capsule fold resection to produce bidirectional flow of synovial fluid between popliteal cyst and knee cavity. Gradually, popliteal cyst will disappear because of the elimination of synovial fluid in popliteal cyst. Communication closure is arthroscopic suture of posteromedial capsular fold suture to close unidirectional flow of synovial fluid [13]. The success rates were 96.7 and $84.6 \%$ in the communication enlargement group [7, 14-18] and communication closure group [12, 19, 20], respectively. Communication enlargement was subgrouped into cystectomy wall resection group $[14,15,17,18]$ and the noncystectomy wall resection group $[7,16]$, for which the success rates were 98.2 and $94.7 \%$, respectively.

We performed partial posteromedial capsulotomy (communication enlargement between articular cavity and popliteal cyst) to produce enough bidirectional flow of synovial fluid (arthroscopic articular cavity internal drainage of popliteal cyst). We also performed cystectomy wall debridement through superior posteromedial portal, which will be useful for popliteal cyst cavity closure through adhesion of medial gastrocnemius tendon and semimembranosus tendon. The treatment effect of technically easily performed communication enlargement is definitive than that of communication closure, 
because communication closure is not definitive to close the communication and more technically difficult to perform.

It is very necessary to perform MRI examinationpreoperatively. MRI is helpful to find not only the communication between knee articular cavity and popliteal cyst but also the underlying intra-articular lesions, such as meniscus lesion, synovitis, and chondral lesion. The popliteal cyst is almost never an isolated pathology in adult knee.

Since a significant association of Baker's cysts with knee joint disorders has been reported, treatment should primarily address articular lesions causing recurrent effusions. Arthroscopic surgery provides an effective treatment in that both the cyst and associated joint disorders can be optimally visualized and accordingly simultaneously treated [13].

An open surgical excision from posterior popliteal fossa cannot be considered as a definitive solution in most popliteal cyst patients. The frequency of recurrence, large popliteal skin scars, and limitation of knee motion after simple open surgical excision from posterior popliteal fossa lead to arthroscopic excision internal drainage of popliteal cyst through posteromedial portal.

Arthroscopic surgery has several benefits: relatively simple, minimally invasive, and early rehabilitation. And most importantly, it is just to excise the posteromedial capsular fold which will enlarge valvular communication to lead to bidirectional flow of synovial fluid, and cystectomy wall can also be arthroscopically resected and performed through superior posteromedial portal.

We did not perform MRI examination after arthroscopic knee articular cavity internal drainage of popliteal cyst and cystectomy cyst wall debridement. The main reason is that the arthroscopic knee cavity internal drainage of popliteal cyst is just to enlarge the communication between knee cavity and popliteal cyst. There is still some remnant fluid in the popliteal cyst. But the pressure inside the popliteal cyst will decrease and the symptoms will disappear. Through cystectomy cyst wall debridement, popliteal cyst cavity will disappear gradually through adhesion of medial gastrocnemius tendon and semimembranosus tendon. So there is no need to perform MRI examination postoperatively.

Our study has proved that arthroscopic internal drainage and cystectomy of popliteal cyst in knee osteoarthritis is effective minimally invasive surgical procedure without recurrence, which was shown by decreased VAS pain score and increased Lysholm knee function score and no recurrence during follow-up period.

\section{Conclusion}

Arthroscopic articular cavity internal drainage and popliteal cystectomy cavity debridement of popliteal cyst and arthroscopic debridement of knee osteoarthritis may eliminate popliteal cyst and improve knee function and is an effective minimally invasive surgery method for the treatment of popliteal cyst without recurrence in knee osteoarthritis patients.

\section{Abbreviations \\ ACRC: Arthritis Clinic \& Research Center; AL: Anterolateral; AM: Anteromedial; JJ: Jun Jiang; LN: Lei Ni; MCL: Medial collateral ligament; PCL: Posterior cruciate ligament; PM: Posteromedial; SPM: Superior posteromedial}

\section{Acknowledgements}

We would like to acknowledge Professor Julio Fernandes for revising our English manuscript. We also would like to acknowledge Dr. Jiang Liu for his assistance for the clinical data collection and statistical analysis.

Funding

There is no funding

Availability of data and materials

Please contact author for data requests.

Authors' contributions

LN conceived of the study and performed these operations. JJ followed up these patients, collected the clinical data, performed the statistical analysis, and drafted the manuscript. Both authors read and approved the final manuscript.

\section{Authors' information}

Lei $\mathrm{Ni}(\mathrm{LN})$ is an arthroscopy specialist and professor in ACRC and is a committee member of an arthroscopy subgroup of Chinese orthopedic society. Jun Jiang $(\mathrm{JJ})$ is also an arthroscopy specialist in ACRC.

Ethics approval and consent to participate

This study was approved by the Ethics Committee of Peking University People Hospital. Informed consent was taken from all patients.

Consent for publication

Not applicable

Competing interests

The authors declare that they have no competing interests.

\section{Publisher's Note}

Springer Nature remains neutral with regard to jurisdictional claims in published maps and institutional affiliations.

Received: 3 August 2017 Accepted: 30 October 2017

Published online: 23 November 2017

\section{References}

1. Fritschy D, Fasel J, Imbert JC, et al. The popliteal cyst. Knee Surg Sports Traumatol Arthrosc. 2006;14:623-8.

2. Lindgren PG, Willen R. Gastrocnemio-semimembranosus bursa and its relation to the knee joint. I Anatomy and histology Acta Radiol Diagn (Stockh). 1977:18:497-512.

3. Rupp S, Seil R, Jochum P, et al. Popliteal cysts in adults. Prevalence, associated intraarticular lesions, and results after arthroscopic treatment. Am J Sports Med. 2002;30:112-5.

4. Liu Y, Xue J, Zhou M, et al. The value of arthroscopic microsurgery for baker cyst accompanied with knee osteoarthritis. The Academic J of 3rd Military Medical University. 2008;30:1408-10.

5. Cho DY, Sec JG, Haam YG. The management of Baker's cyst (the new surgical technique for the prevention of recurrence). J Korean Orthop Assoc. 1994;29:288-93.

6. Lindgren PG. Gastrocnemio-semimembranosus bursa and its relation to the knee joint. III. Pressure measurements in joint and bursa. Acta Radiol Diagn. 1978;19:377-88. 
7. Sansone V, De Ponti A. Arthroscopic treatment of popliteal cyst and associated intra-articular knee disorders in adults. Arthroscopy. 1999;15:368-72.

8. Field JR, Franklin PD, Kustan J. Popliteal cysts: a reassessment using magnetic resonance imaging. Skelet Radiol. 1991;20:433-5.

9. Johnson LL, van Dyk GE, Johnson CA, Bays BM, Gully SM. The popliteal bursa (Baker's cyst): an arthroscopic perspective and the epidemiology. Arthroscopy. 1997;13:66-72.

10. Labropoulos N, Shifrin DA, Paxinos O. New insights into the development of popliteal cyst. Br J Surg. 2004;91:1313-8.

11. Marti-Bonmati L, Molla E, Dosda R, et al. MR imaging of baker cystsprevalence and relation to internal derangements of the knee. MAGMA. 2000;10:205-10.

12. Takahashi M, Nagano A. Arthroscopic treatment of popliteal cyst and visualization of its cavity through the posterior portal of the knee. Arthroscopy. 2005;21:638.

13. Calvisi V, Lupparelli S, Giuliani P. Arthroscopic all-inside suture of symptomatic Baker's cysts: a technical option for surgical treatment in adults. Knee Surg Sports Traumatol Arthrosc. 2007;15:1452-60.

14. Cho JH. Clinical results of direct arthroscopic excision of popliteal cyst using a posteromedial portal. Knee Surg Relat Res. 2012;24:235-40.

15. Ahn JH, Lee SH, Yoo JC, Chang MJ, Park YS. Arthroscopic treatment of popliteal cysts: clinical and magnetic resonance imaging results. Arthroscopy. 2010;26:1340-7.

16. Ohishi T, Takahashi M, Suzuki D, Fujita T, Yamamoto K, Ushirozako H, et al. Treatment of popliteal cysts via arthroscopic enlargement of unidirectional valvular slits. Mod Rheumatol. 2015;25:772-8.

17. Yinghui $H$, Shiyi $C$, Weitao $Z$, et al. 35 cases report of arthroscopic treatment of popliteal cyst. Chinese sports medicine Journal. 2006;25:279-301.

18. Ko SH, Ahn JH. Popliteal cystoscopic excisional debridement and removal of capsular fold of valvular mechanism of large recurrent popliteal cyst. Arthroscopy. 2004;20:37-44.

19. Rauschning W. Popliteal cysts (Baker's cysts) in adults. II. Capsuloplasty with and without a pedicle graft. Acta Orthop Scand. 1980;51:547-55.

20. Hughston JC, Baker CL, Mello W. Popliteal cyst: a surgical approach. Orthopedics. 1991;14:147-50

\section{Submit your next manuscript to BioMed Central and we will help you at every step:}

- We accept pre-submission inquiries

- Our selector tool helps you to find the most relevant journal

- We provide round the clock customer support

- Convenient online submission

- Thorough peer review

- Inclusion in PubMed and all major indexing services

- Maximum visibility for your research

Submit your manuscript at www.biomedcentral.com/submit 